International Journal of Engineering \& Technology, 7 (4.5)(2018) $179-181$
International Journal of Engineering \& Technology
SPC
Website: www.sciencepubco.com/index.php/IJET
Research paper

\title{
Modified Turbo and SDROM Method for Speech Processing for Cochlear Implants
}

\author{
Rohini S.Hallikar ${ }^{1}$, M Uttarakumari ${ }^{2}$, Padmaraju $\mathrm{K}^{3}$ and Yashas $\mathrm{D}^{4}$ \\ ${ }^{I}$ R.V.C.E.,Bengaluru,Karnataka, India \\ ${ }^{2}$ R.V.C.E.,Bengaluru, Karnataka, India \\ ${ }^{3}$ J.N.T.U.Kakinada, Andhra Pradesh, India \\ ${ }^{4}$ R.V.C.E.,Bengaluru,Karnataka, India \\ *Corresponding author E-mail:rohinish@rvce.edu.in
}

\begin{abstract}
A performance comparison of Signal Dependent Rank Order Mean (SDROM) method of speech signal enhancement with a speech enhancement method which makes use of a Turbo combination and SDROM filter referred to as modified Turbo and SDROM technique is made in this paper. Normally, speech signals are used as inputs to a cochlear implant signal processing unit.

Sounds are corrupted by different noises such as AWGN, Impulsive noise and babble. The results are evaluated in terms of enhancements evaluations done by basically three parameters namely correlation coefficient, log spectral distortion (LSD) and segmental signal to noise ratio(SSNR). These parameters are calculated between the processed and the clean signals.. Results prove the superior performance of the new method especially for AWGN corrupted speech.
\end{abstract}

Keywords:. Cochlear Implant; Signal Dependent Rank Order Mean (SDROM),;Impulsive,;AWGN and Babble

\section{Introduction}

Designing a speech processor for cochlear prosthesis is a multifaceted task. Such processors at a very basic level must extract or preserve from speech those parameters which are very crucial for intelligibility. Also it is necessary for the speech processor to encode those parameters for electrical stimulation of the auditory nerve. [1]A sense of sound could be provided to people who are deaf or profoundly hearing impaired by using a cochlear implant. Majority of the users could converse over the telephone for everyday communications. [2]Profoundly deaf people were restored partial hearing successfully using Cochlear Implants. Its success could be seen as combined efforts of scientists from interdisplinary research areas such as bioengineering, physiology, signal processing, otolaryngology and speech sciences. For developing different techniques so as to derive electrical stimulus from speech signals, signal processing played a significant role. [3] CI provides two opportunities such as studying speech perception and investigation of perceptual limits of the auditory system. We could investigate as to how limited intensity and spectral resolution affects speech understanding. Also we could pose questions as to understand speech what is the number of channels required? The answer to this question may be used to design very low rate speech coders. [4]

To avoid interplay between channels Wilson first proposed the CIS strategy by introducing asynchronous and alternate pulses. CIS processing is explained as follows.

1) Initially Speech signal is divided into 4 to 8 channels which is subjected to preemphasis.
2) Processing each channel with full wave rectification and low pass filtering next envelope is obtained.

3) Compression of envelope is followed. It may be logarithmically compressed or square rate afterward.

4) Generation of simulating pulse of every electrode happens by modulation of the envelopes temporarily by alternating biphasic pulse sequence. [5]

Another well-known signal processing strategy is named as the Advanced Combinational Encoder (ACE). In this technique the input signal is sampled at $16 \mathrm{kHz}$. Using window frames of $8 \mathrm{~ms}$ or a number $\mathrm{L}$ of 128 samples is used for analysis of the input. To obtain each frame spectral decomposition an M band filterbank is used. $\mathrm{M}$ is number of implanted electrodes. Implants of the patients tested are configured with only 20 electrodes hence a 20 band assembly is considered. Hence provision of $\mathrm{L}=128$ spectral coefficients is possible. Using FFT symmetry property for real input samples, the second half of the spectrum can be discarded without any information loss. Envelope detector uses the first 64 bins and rearranges them in a non-uniform way closer to critical band portion by the human cochlea. [6]

SDROM was successfully been used for removing impulsive noise from images. It is relatively fast, robust and simple. The likelihood is estimated by the algorithm. Relative to the neighbouring samples a corrupt sample under observation is said to be corrupt. Based on neighbouring samples there is a replacement for a sample detected as corrupted.

For filtering images SDROM method which is variant of the original algorithm is used. 2D sample window is used in image resto- 
ration. Size of window was 3 by 3 . A 1-D sliding window is used in this method. Window of size five is used. Refer figure 1. [7]

$$
\begin{array}{|l|l|l|l|l|}
\hline X_{n-2} & X_{n-1} & X_{n} & X_{n-1} & X_{n+2} \\
\hline
\end{array}
$$

Fig1: Filter Window

Signal enhancement is done in the Turbo filter by considering two filters, one a time domain filter and second using a frequency domain filter. The first one is a Kalman filter and the second is the DRNL filter.

Turbo iteration maximizes the benefits of frequency and time domain speech enhancement techniques.

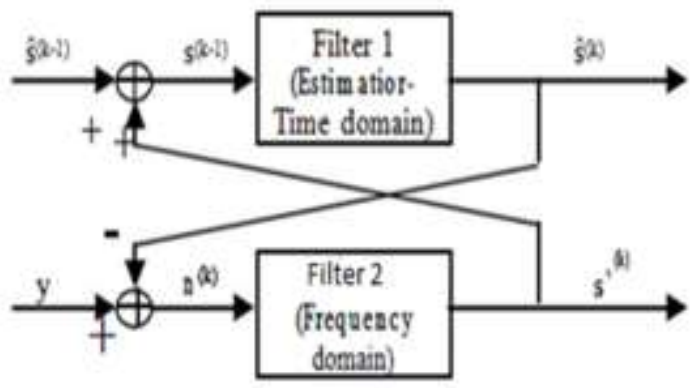

Fig 2: Signal Enhancement using Turbo Method

Turbo architecture flow is as shown in Figure 3 which explains the processes which occurs when the turbo method is operated.

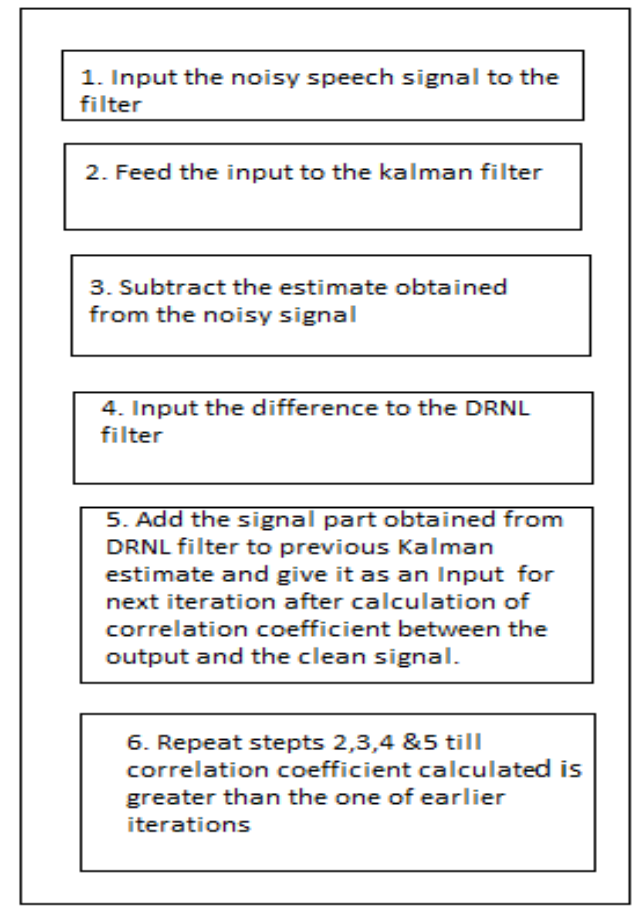

Fig 3: Flow for Turbo architecture.

Kalman filter is a recursive algorithm which is based on the use of state space technique. It consists of two steps.

Namely 1) Prediction and 2) Correction.

A dynamic model is used to predict the state. Using the observation model correction is made so as to minimize error covariance of estimator. Nonlinear auditory frequency selectivity relating to physiological and psychophysical phenomenon was reproduced by direct resonance nonlinear (DRNL) filter. With change with the amplitude of its input signal DRNL filters tuning, best frequency, gain and phase characteristics changes. There is alteration in the spectral content of the input waveform due to alteration by nonlinear filters; also they do not have proper transfer functions. An approximate transfer function of the DRNL filter is possible as one of its components is time invariant and linear [8].

\section{Methodology}

Input signals sp01 is fed to the two filter stages viz, SDROM followed by Turbo to obtain the output signal for which Correlation Coefficient, LSD and SSNR is computed. This way of processing is referred to as Modified Turbo and SDROM technique.

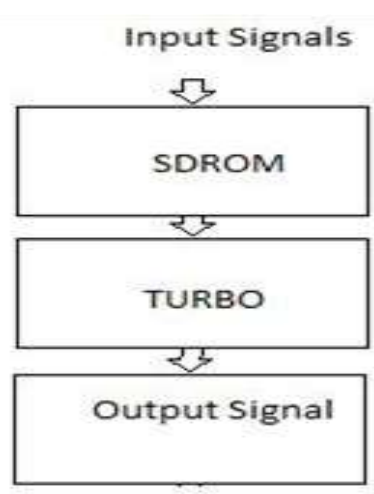

Fig 4: Modified Turbo and SDROM technique

\section{Results}

Modified Turbo and Turbo algorithms for different

SNR's

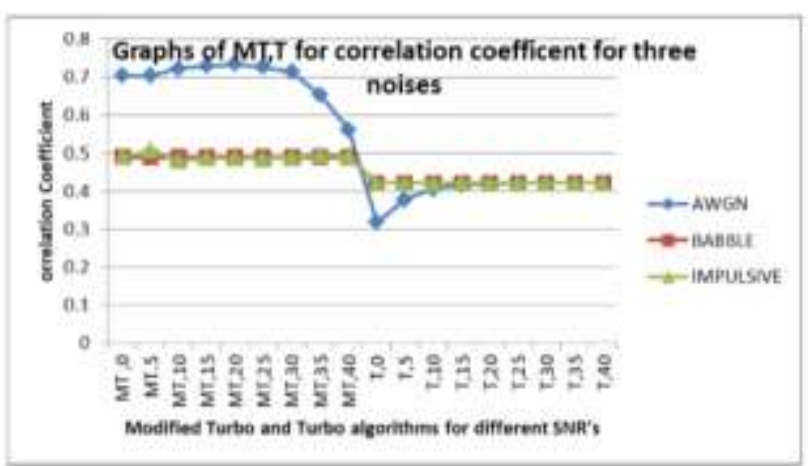

Fig 5: Plots of Modified Turbo and SDROM (MT) and Turbo (T) techniques for correlation coefficient at different SNR are for three noise types.

From the figure 5 it is clear that Modified Turbo and SDROM is much superior compared to Turbo technique.

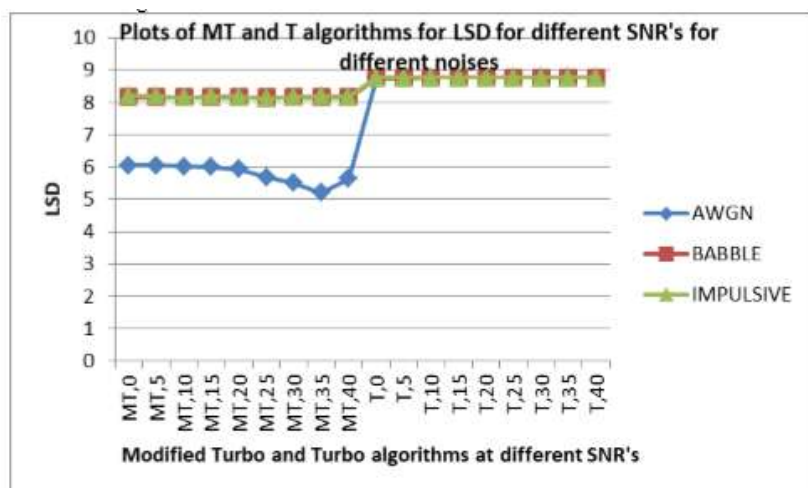

Fig 6: Plots of Modified Turbo and SDROM (MT) and Turbo (T) techniques for LSD at different SNR's for three noise types. 
Figure 6 shows that LSD measurements for Modified Turbo and SDROM techniques are lower as compared to Turbo technique reflecting upon the superiority of Modified Turbo and SDROM technique.

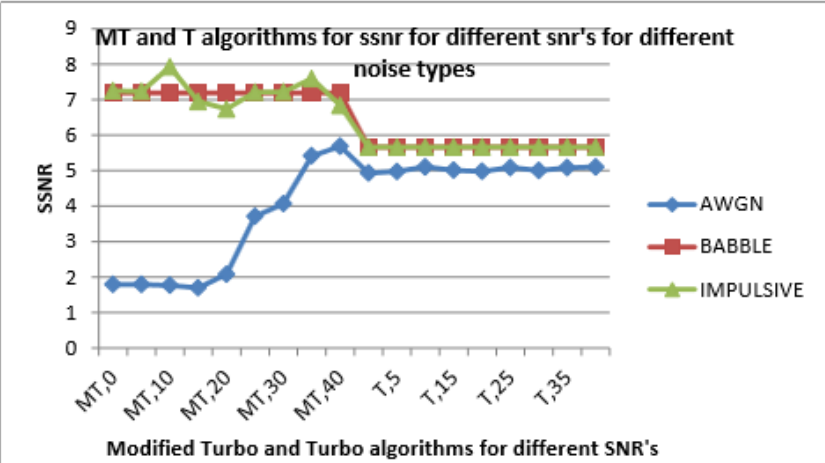

Fig 7: Plots of Modified Turbo and SDROM (MT) and Turbo (T) techniques for SSNR at different SNR's for three noise types.

Figure 7 shows that SSNR computations are higher for Babble and Impulsive signals in the case of Modified Turbo and SDROM technique as compared to Turbo technique.

\section{Conclusion}

According to the results proposed and from figures of 5, 6 and 7 it is clear that Modified Turbo and SDROM technique is superior to Turbo technique and provides good enhancement to the signals irrespective of the noise type.

\section{References}

[1] Wilson BS, Finley CC, Lawson DT, Wolford RD. Speech processors for cochlear prostheses. Proceedings of the IEEE. 1988 Sep;76(9):1143-54.

[2] An SK, Park SI, Jun SB, Lee CJ, Byun KM, Sung JH, Wilson BS, Rebscher SJ, Oh SH, Kim SJ. Design for a simplified cochlear implant system. IEEE Transactions on Biomedical Engineering. 2007 Jun;54(6):973-82.

[3] Loizou PC. Signal processing for cochlear prosthesis: a tutorial review. In Circuits and Systems, 1997. Proceedings of the 40th Midwest Symposium on 1997 Aug 3 (Vol. 2, pp. 881-885). IEEE.

[4] Loizou PC. Signal processing for cochlear implants and low-rate speech coding. In Speech Coding, 2000. Proceedings. 2000 IEEE Workshop on 2000 (p. 68). IEEE.

[5] Wang L, Zhou L, Xu Y, Xiao Z. Computer simulation of multichannel CIS strategy for cochlear implant. In Bioinformatics and Biomedical Engineering, 2009. ICBBE 2009. 3rd International Conference on 2009 Jun 11 (pp. 1-4). IEEE

[6] Albalate A, Nogueira W, Edler B, Buchner A. Signal analysis by using adaptive filterbanks in cochlear implants. In Biomedical Circuits and Systems Conference, 2006. BioCAS 2006. IEEE 2006 Nov 29 (pp. 210-213). IEEE.

[7] Abreu E, Mitra SK. A signal-dependent rank ordered mean (SDROM) filter-a new approach for removal of impulses from highly corrupted images. In Acoustics, Speech, and Signal Processing, 1995. ICASSP-95., 1995 International Conference on 1995 May 9 (Vol. 4, pp. 2371-2374). IEEE.

[8] Hallikar RS, Kulkarni S, Uttarakumari M, Padmaraju K. AWGN and impulsive noise removal by turbo audio enhancement technique. In Control, Instrumentation, Communication and Computational Technologies (ICCICCT), 2014 International Conference on 2014 Jul 10 (pp. 1254-1259). IEEE. 